\title{
Yoga and movement re-education for the treatment of idiopathic scoliosis
}

\author{
Marcia Monroe \\ From 7th International Conference on Conservative Management of Spinal Deformities \\ Montreal, Canada. 20-22 May 2010
}

\section{Summary}

Yoga and Movement Reeducation for the treatment of idiopathic scoliosis: case study of a 46-year-old.

\section{Objective}

To describe the use of Iyengar Yoga therapy and movement reeducation techniques in the management of a 46-year-old woman with idiopathic scoliosis.

\section{Introduction}

Idiopathic scoliosis is the most common form of scoliosis. Adolescent Idiopathic Scoliosis (AIS), which develops in young adults around the onset of puberty, represents approximately $80 \%$ of idiopathic scoliosis cases. This is a case presentation of a 46-year-old woman who was diagnosed with idiopathic scoliosis and recommended as a candidate for spinal fusion during her early adolescent years. She instead chose to work with conservative therapy sessions for the period of one year. She then opted to practice somatic movement reeducation techniques and yoga, mainly Body-Mind Centering $^{\mathrm{TM}}$, The Feldenkrais Method ${ }^{\mathrm{Tm}}$, swimming, and Iyengar Yoga.

\section{Clinical features}

Head and neck were tilted to one side, shoulders were not level, a raised prominent hip and rib cage were at different heights, and the waist was uneven, leaning the body to one side. In addition, early signs of scoliosis were shown in childhood: everted or sunken sternum, inversion of right foot, and instability of both calcaneous bones.

\section{Prognosis}

Continued growth of the curvature, affecting equilibrium and gait. Expectations of a limited functional life with episodes of acute pain and major health implications.

\section{Outcome}

The subject has been studying and teaching Iyengar Yoga, reinforced with movement reeducation practices, on a daily basis. The yoga regimen shown in this presentation, has helped the patient to stabilize weak muscles; enhance the range of respiration; increase postural tone in standing, sitting, and lying; and strengthen and lengthen the spine through specific postures used as traction. Movement reeducation based on the integration of concepts of bio-mechanics and motor development has improved the subject's proprioception, sensibility, and awareness of functional movement. She is now 46-years-old and pain-free, living a functional and active life.

\section{Conclusion}

A regimen of Iyengar Yoga combined with movement reeducation based on perceptual and motor development from Body-Mind Centering and The Feldenkrais Method may be very useful in reducing pain and improving the well-being and functional abilities in idiopathic scoliosis regardless of age.

Published: 10 September 2010

doi:10.1186/1748-7161-5-S1-O24

Cite this article as: Monroe: Yoga and movement re-education for the treatment of idiopathic scoliosis. Scoliosis 2010 5(Suppl 1):O24. 